\title{
Prussian-Blue-Doped Super-Activated Carbon as a High Performance Supercapacitor Electrode Material
}

Qingyong Wang ${ }^{1,2}$, Yongjin Zou ${ }^{1,2,3, *}$, Cuili Xiang ${ }^{1,2}$, Hailiang Chu ${ }^{1,2}$, Shusheng Liu ${ }^{1,2, *}$, Erhu Yan ${ }^{1,2}$, Fen $\mathrm{Xu}^{1,2}$, Lixian Sun ${ }^{1,2}$, Chengying Tang ${ }^{1,2}$

${ }^{1}$ Guangxi Key Laboratory of Information Materials, Guilin University of Electronic Technology, Guilin 541004, P.R. China

${ }^{2}$ Guangxi Collaborative Innovation Center of Structure and Property for New Energy Materials, Guilin 541004, P.R. China

${ }^{3}$ Guangxi Experiment Center of Information Science, Guilin University of Electronic Technology, Guilin 541004, P.R. China

*E-mail: zouy@guet.edu.cn, 25245195@qq.com

doi: $10.20964 / 2016.07 .98$

Received: 11 April 2016 / Accepted: 1 May 2016 / Published: 4 June 2016

A Prussian-blue-doped super activated carbon (PB/SAC) nanocomposite was prepared by a simple reduction method under mild condition. Scanning electron microscopy, X-ray diffraction, Fouriertransform infrared spectroscopy, and transmission electron microscopy experiments revealed that PB nanoparticles were incorporated in the pores of the SAC. Cyclic voltammetry measurements indicated that the combination of PB and SAC significantly enhanced the capacity of the composite. The hybrid $\mathrm{PB} / \mathrm{SAC}$ electrode yielded the maximum capacitance performance with a specific capacitance up to $263.7 \mathrm{~F} \mathrm{~g}^{-1}$ at a current density of $5 \mathrm{~A} \mathrm{~g} \mathrm{~g}^{-1}$. The rational combination of these two materials provides the devices with an extended voltage window of $0.8 \mathrm{~V}$ in acid solution. Cyclic stability measurements indicated that the specific capacitance of the PB/SAC nanocomposite electrode could retain $94.8 \%$ of its initial value over 1500 charge/discharge cycles. More significantly, the supercapacitors were designed to be low in cost and environmentally benign, and are therefore highly suitable for future energy storage systems.

Keywords: Supercapacitor; Super activated carbon; Prussian blue; Nanocomposite

\section{FULL TEXT}

(C) 2016 The Authors. Published by ESG (www.electrochemsci.org). This article is an open access article distributed under the terms and conditions of the Creative Commons Attribution license (http://creativecommons.org/licenses/by/4.0/). 